\section{Non oncologic elevated levels of chromogranin A in a symptomatic patient without a carcinoid tumor}

\author{
Juan Camilo Duque, ${ }^{1}$ Luis Fernando \\ Pineda, ${ }^{2}$ Tatiana Lopez ${ }^{3}$ \\ 1 DeWitt Daughtry Family Department \\ of Surgery, Leonard M. Miller School \\ of Medicine, University of Miami, FL, \\ USA; 2 Centro de Enfermedades \\ Digestivas, Bogotá, Colombia; ${ }^{3}$ Division \\ of Endocrinology, National Cancer \\ Institute, Bogota, Colombia
}

\section{Abstract}

The diagnosis of neuroendocrine tumors requires a highly suspicious level due to its variable symptoms and clinical presentation. Early diagnosis, prompt and appropriate treatments are clues to success preventing the eventual disease progression and the devastating consequences of the carcinoid syndrome. Measurement of the chromogranin A, plays an important role in the diagnosis of carcinoid tumors, during the clinical evolution and even more to evaluate the treatment response to somatostatin analogues. However, in some situations like our case, the serum levels evaluation could be a confounding factor imitating the clinical scenario of the disease especially when the chromogranin level is elevated but not in the typical oncologic range and moreover when the symptoms magnitude is really convincing and similar to the tumor disease instances.

\section{Case Report}

A 58-year-old Hispanic woman with non-past medical history and negative history of risk factors presented to the emergency department with one-day history of lower extremity edema, shortness of breath and tachycardia. She has a history of mammary prosthesis replacement 28 days ago. An initial diagnosis of pulmonary embolism was made; a pulmonary angio computed tomography (CT) was performed showing no vascular occlusion. Transthoracic echocardiogram has shown normal EF of $60 \%$ and no valvular disease. Coronary pharmacologic perfusion echocardiogram showed normal heart function and finally renal function was normal. The patient was treated for acute heart failure and discharged with ambulatory control. Six months later, upper gastric endoscopy was performed evi- dencing multiple polyps. Immediately after the procedure, the patient presented an episode of upper body flushing and an intense diffuse dull abdominal pain without rebound and tenderness. She concern that she had experienced explosive episodes of non bloody diarrhea, self limited episodes of dry cough, frequent episodes of flushing in the upper trunk and face with profuse sweating and intense pruritus lasting 15 minutes associated with significant unintentional weight loss over the last 6 month. Further workup included negative brain MRI, negative chest CT and abdominal MRI that only showed multiple benign liver cysts, (Figure 1) pancreatic and rectal endosonography were also normal. Somatostatin receptor scintigraphy (SRS) with Indium-111-DPA-octreotide was negative, capsule endoscopy negative for masses, 24-hour urine level of 5-HIAA appeared borderline 9.8 $\mathrm{mg} / 24 \mathrm{hr}$ (range levels 1.9-10.0 mg/24 hr) and plasma chromogranin A were above the reference range $92.0 \mathrm{ng} / \mathrm{mL}$ (range levels 1.9-15.0 $\mathrm{ng} / \mathrm{mL}$ by electrochemilumiscent method) (Table 1). An endoscopic polypectomy was done and histopathology reported hyperplasic polyps. Three months later, due to the persistent and more intense symptoms, a SRS with Indium-111-DPA-octreotide was repeated showing positive reactivity at the epigastric area and chromogranin A control persists elevated. The patient was placed on monthly intramuscular octreotide with dramatic improvement of the symptoms and chromogranin A levels. Nine months later a SRS was repeated as a control denying abnormal capitation (Figure 2). The octreotide was discontinued but two months later after a new upper endoscopic control the patient reported general discomfort given by more intense and longer flushing episodes associated with hypogastric dull pain radiated to lower back, which did not relieved with multiple analgesic and antispasmodic medications that stared one day after the endoscopy. Interrogating about the pain and discomfort, patient denote that she has been continued losing weight unintentionally and emphasized that during this period of medication withdraw had an episodes of vertigo and symptoms of low heart output given by tinnitus, profuse sweet, cold extremities and tachycardia. Double contrast abdomino-pelvic CT revealed no abnormality. Assessment of chromogranin A showed frank increase 150.20 $\mathrm{ng} / \mathrm{mL}$ (range levels $1.9-15.0 \mathrm{ng} / \mathrm{mL}$ by electrochemilumiscent method). The patient was placed on subcutaneous Lanreotide with symptomatic and blood chemestry improvement.

\section{Discussion}

Neuroendocrine tumors (NET) are hetero-
Correspondence: Juan Camilo Duque, DeWitt Daughtry Family Department of Surgery, Leonard M. Miller School of Medicine, University of Miami Miller School of Medicine, 1600 NW 10th Avenue, Miami, 33136 FL, USA.

Tel. +1.786.4522127.

E-mail: j.duque1@med.miami.edu

Key words: neuroendocrine tumors, chromogranin A, somatostatin analogues.

Contributions: JCD, ambulatory controls; LFP, endoscopic procedures, discussion; TL, patient follow up regulating the hormonal treatment.

Conflict of interests: the authors declare no potential conflict of interests.

Received for publication: 29 April 2013.

Revision received: 28 May 2013.

Accepted for publication: 4 June 2013.

This work is licensed under a Creative Commons Attribution NonCommercial 3.0 License (CC BYNC 3.0)

(c) Copyright J.C. Duque et al., 2013

Licensee PAGEPress, Italy

Endocrinology Studies 2013; 3:e7

doi:10.4081/es.2013.e7

geneous groups of tumors arising from neuroendocrine cells showing distinct functional and biological behavior depending on location, tumor size, and clinical symptoms. ${ }^{1}$ Carcinoid tumors derives from enterochromaffin cells also known as Kulchitsky cells which are distributed everywhere around the human body.2 This cell population is mostly predominant along the gastro intestinal tract, in the urogenital tract and lungs specifically as part of the bronchial epithelium. ${ }^{3}$ The diagnosis of this type of tumors is different in each individual depending on the embryologic origin of the tumor, which could show different clinical manifestations, blood chemical parameters and distinctive morphology and histology. Particularly carcinoid tumors secrete serotonin and its metabolites however, they are able to secrete different compounds like bradykinin or other vasoactive elements depending on the histology and their anatomical location. ${ }^{4}$ In normal patients, approximately $99 \%$ of tryptophan from the normal diet is used for the nicotinic acid synthesis and around $1 \%$ is metabolized into 5 hydroxytryptamine (5-HT). Patients diagnosed with carcinoid tumors present a shift toward the production of 5-HT and eventually 5-Hydroxyindoleacetic acid (5-HIAA) that is the main metabolite of serotonin. The rising of this products and the lack of metabolization of them are responsible for the typical signs and symptoms in this group of patients, ${ }^{3}$ therefore in early stages of disease the patients may 
present nonspecific symptoms that often led to misdiagnose. The typical described symptoms and the common signs seen in these patients include bronchospasm which is mediated by serotonin or bradykinin effect on the bronchial muscle, diarrhea which is mediated by serotonin, cutaneous flushing that is the most frequent described uncomfortable symptom and right-sided valvular heart lesions that is probably is the worst complication which is mediated by serotonin. ${ }^{4}$ High percentage of the NET are characterized because of their benign behavior however, all of them are prone to turn malignant and are able to metastases easily specially in the malignant forms worsen the patient prognosis also develop late recurrences after successful treatments or turn rapidly into an aggressive course. When the diagnosis is made in the late stages the probability of metastasis is higher and these usually present at pancreas (76\%) follow by the colon (71\%). ${ }^{3}$

The available literature showed an increasing incidence of this kind of tumors from 1.09 cases per 100,000 in 1973 to 5.25 per 100,000 in 2004.,6 The exact reason for this increase is not well understood, however, it might be related to increase of awareness among trained physicians as well as better diagnosis tools and available treatments. ${ }^{2}$ The etiology of carcinoid tumors remains unknown however, with the molecular advances the literature reports shown a non-relationship with the common oncogenes such as ras, myc, fos, jun and $s r c$ as well with the tumor suppressor genes p53 and retinoblastoma except in the more atypical forms. ${ }^{3}$

The localization in general is predictable due to the common rising in some blood chemistry test; all of them have the capability to produce a hormonal misbalance sharing the blood elevation of chromogranin A ( $\mathrm{CgA})$ as a common point. ${ }^{2}$ According to this evidence the presentation and findings can change based on the embryonic origin of the tumor; the foregut lesions account for $20-25 \%$ of all carcinoid tumors in which normally the primary lesions are found in the lung, thymus, stomach, or proximal duodenum with few cases along the hepatobiliary tract or pancreas. ${ }^{7}$ There are usually associated with low serotonin levels but also they are able to produce serotonin precursors, histamine, corticotropin releasing hormone, adrenocorticotropic hormone (ACTH), gastrin, and CgA. Moreover midgut carcinoid tumors that are considered the most prevalent variation account for 40 $50 \%$ of cases. Classically they are found at the distal duodenum, jejunum, ileum, appendix, or proximal colon. However, the appendix is the most common location. They are characterized

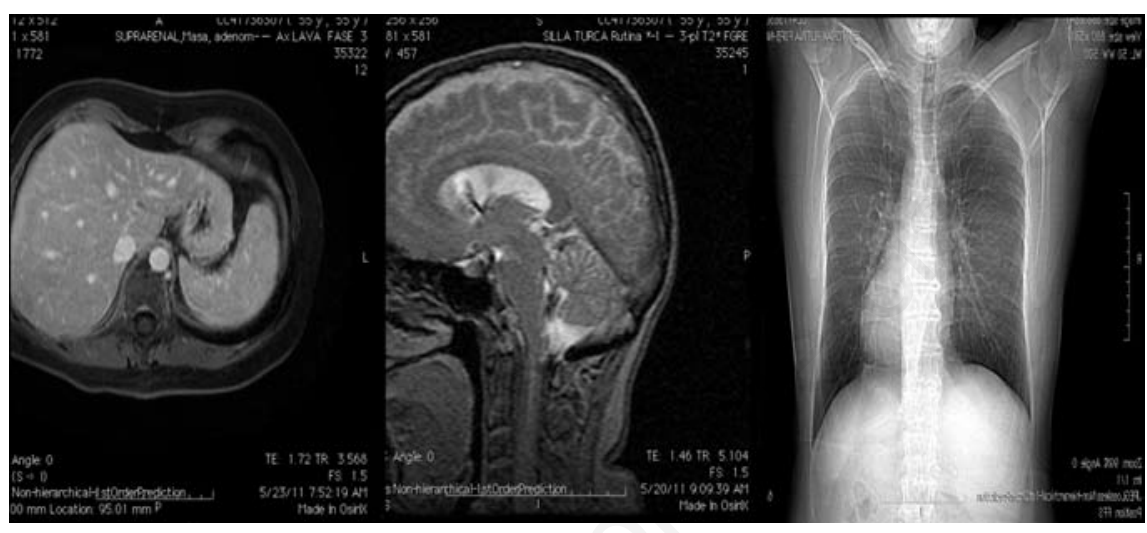

Figure 1. Negative brain magnetic resonance imaging, negative chest computed tomography and abdominal magnetic resonance imaging showing multiple benign liver cysts.
Table 1. Chromogranin and 5-HIAA history.

\begin{tabular}{lc} 
Levels & Date \\
& Chromogranin \\
$92.0 \mathrm{ng} / \mathrm{mL}$ & $17 / 03 / 2011$ \\
$72.0 \mathrm{ng} / \mathrm{mL}$ & $12 / 10 / 2011$ \\
\hline $86.40 \mathrm{ng} / \mathrm{mL}$ & $25 / 02 / 2012$ \\
$20.60 \mathrm{ng} / \mathrm{mL}$ & $11 / 05 / 2012$ \\
\hline $82.80 \mathrm{ng} / \mathrm{mL}$ & $22 / 09 / 2012$ \\
$66.30 \mathrm{ng} / \mathrm{mL}$ & $08 / 01 / 2013$ \\
\hline $150.20 \mathrm{ng} / \mathrm{mL}$ & $18 / 03 / 2013$ \\
$103.50 \mathrm{ng} / \mathrm{mL}$ & $25 / 04 / 2013$ \\
\hline & \\
\hline $9.8 \mathrm{mg} / 24 \mathrm{hr}$ & $02 / 12 / 2011$ \\
$11.0 \mathrm{mg} / 24 \mathrm{hr}$ & $25 / 04 / 2013$ \\
\hline
\end{tabular}

Range reference: 1.9-15.0 (Chromogranin); 2.0-10.0 (5-HIAA).

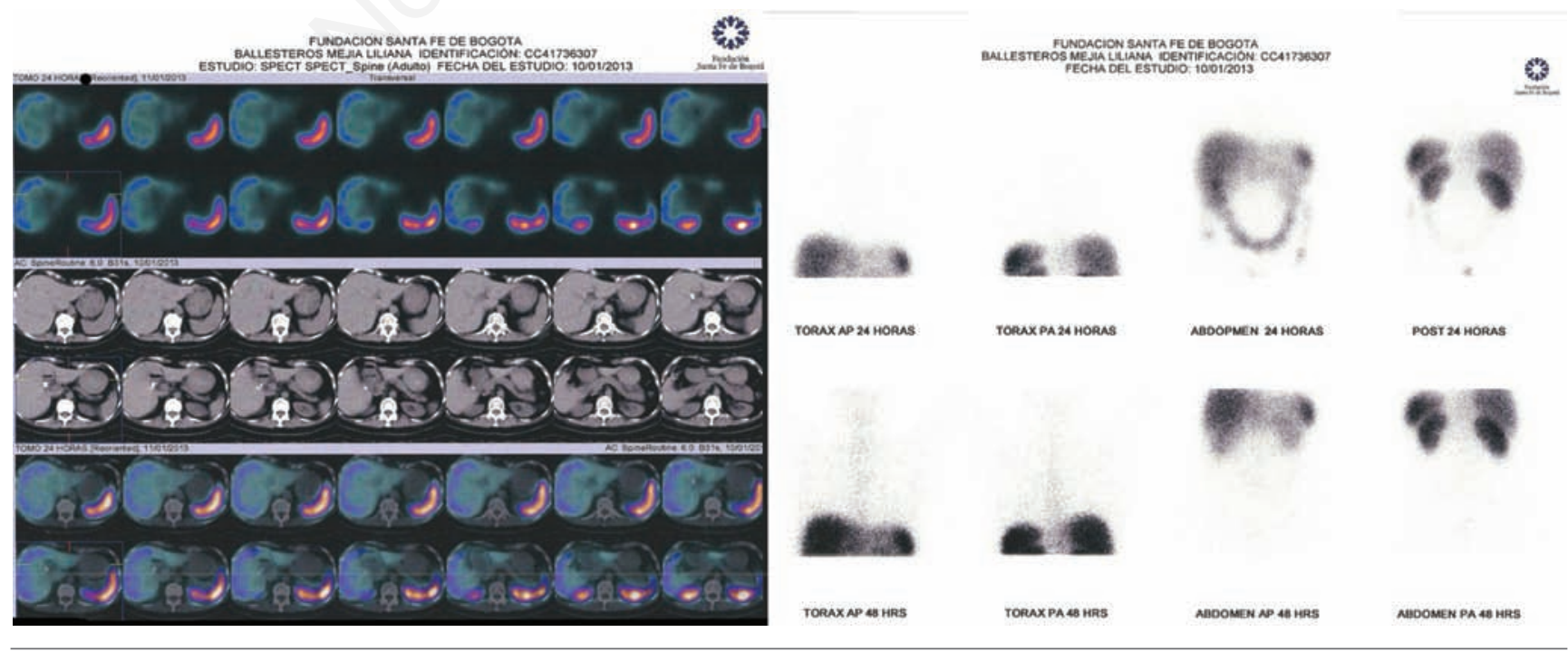

Figure 2. Somatostatin receptor scintigraphy. 
because they secrete 5-HT, bradykinins, tachykinins, histamine, substance P, ACTH, and also CgA. Finally the less common form is originated from the hindgut accounting for $15 \%$ of cases and commonly seen at the distal part of the colon and rectum. ${ }^{4}$ Hindgut tumors mainly secrete hormones like pancreatic polypeptide, somatostatin, and chromogranin A. ${ }^{2}$ As a diagnostic instrument, tumor serum markers represent an essential mechanism to make the diagnosis, playing an important role as a prognostic factor or as a control to evaluate the treatment response. 8,9 These serum markers are a heterogeneous group of molecules that normally are present in small blood concentrations in healthy individuals. In patients with NET, their blood concentration increase dramatically due to the ability of the enterochromaffin cells to increase the exocytosis and also the induction of bioactive substances by other cells. In patients diagnosed with NET, the main evaluated serum marker is by far the chromogranin A. It is an acidic glycoprotein widely expressed by neuroendocrine cells and constitutes one of the most abundant components of secretory granules with a reported sensitivity of $71.3 \%$ and $84 \%$ and specificity between $71 \%$ and $85 \% .{ }^{10}$ High $\mathrm{CgA}$ levels have been demonstrated in the serum or plasma of patients with different types of NET but represent the most important factor in carcinoid tumor. ${ }^{11-13}$ Specifically in the case of carcinoid tumors, $\mathrm{CgA}$ is highly remarkable; it has been shown to be an independent prognostic factor because it correlates with the tumor load and illustrates the biological activity potency of the tumor. ${ }^{7,14}$ However, other non-oncologic medical conditions that are able to raise the blood CgA levels develop several clinical symptoms that can mimic a NET. This increment is commonly seen in some medical conditions like impaired kidney function or even more common in individuals with chronic atrophic gastritis treated with proton pump inhibitors medications. ${ }^{7}$ Nowadays, diagnostic imaging of this kind of tumors appears more accurate, reliable and is useful for following up the patient condition. Numerous imaging modalities are applied for the diagnosis, characterization of the tumor and for the clinical staging.

The most used are CT and MRI that are the first step and primary imaging modalities used to establish the possible diagnosis and the anatomical location of the tumors. On the other hand, exist ultrasound techniques, which are getting more importance due to their clear and direct visualization of the lesion during the procedure; the most recognized is the endosonography alone or guided by endoscopy this technique is essential to calculate the depth, infiltration and the compromise of adjacent tissues. Finally, nuclear medicine plays a pivotal role in the diagnostic tools with the applying of Somatostatin receptor scintigraphy (SRS) with Indium-111-DPAoctreotide and the positron emission tomography (PET)-CT which are consider the gold standard technique in NET. Moreover, these functional imaging techniques have multiple utilities and are not only applied for the tumor diagnosis. They are useful to study the tumor compromise and to predict the medical therapeutic response.5,15 As a treatment, the resection in nonmetastized tumors is the cornerstone strategy however, in symptomatic patients in whom the tumor cannot removed due to the metastasis or due to technical reasons, somatostatin analogs are the treatment of choice. It has been reported that these medication responses shown close to $100 \%$ of symptomatic recovery with clear benefits over the few side effects.16 Lanreotide and octreotide are the most common prescribed active compounds that act through a family of five different G-protein receptors to exert a variety of functions, including the inhibition of endocrine and exocrine active secretions that occur not only in the tumoral cells. As well it has been reported in the literature the property to decrease the tumoral growing in $4 \%$ to $10 \%$ of the treated patients. 6,16

\section{Conclusions}

Serum chromogranin A plays an important role as a diagnostic and follow up tool in patients diagnosed with NET especially in cases of carcinoid tumors due to the origin of the marker. However, in situations in which the increases serum levels are not in neoplastic ranges could generate positive confounding symptoms like in our case. We found that after carefully and detailed study, the presence of a tumor was ruled out however, it is clear that our patient has an increase levels of Serum chromogranin A and a 5-Hydroxyindoleacetic acid (5-HIAA) characteristically in the upper range or slightly elevated. These elevations developed signs and symptoms that are characteristic in patients with carcinoid tumors in whom the Chromogranin A levels are extremely high. In our case we highlighted the benefits of the treatment with somatostatin analogues in the absence of the tumor decreasing the serum levels of chromogranin A and showing clear improvement in the patient condition improving her quality of life. Highly remarkable in our case the multidisciplinary team work that is probably the pivotal point in the way of how to treat and handle this difficult case very uncommon in our location due to the multiple and difficult symptoms.

\section{References}

1. Niederle MB, Niederle B. Diagnosis and treatment of gastroenteropatic neuroendocrine tumors: current data on a prospectively collected, respectively analyzed clinical multicenter investigation. Oncologist 2011;16:60213.

2. Scarsbrook A, Ganeshan A, Statham J, et al. Anatomic and functional imaging of metastatic carcinoid tumors. Radiographics 2007;27: 455-76.

3. Kaltsas GA, Besser GM, Grossman AB. The diagnosis and medical management of advanced neuroendocrine tumors. Endocr Rev 2004;25:458-511.

4. Aggarwal G, Obideen K, Wehbi M. Carcinoid tumors: what should increase our suspicion? Cleve Clin J Med 2008;75: 849-55.

5. Oberg K. Neuroendocrine tumors: recent progress in diagnosis and treatment. Endocr Relat Cancer 2011;18 (Suppl 1):E3-6.

6. Oberg K, Casanovas 0, Castano J, et al. Molecular pathogenesis of neuroendocrine tumors: implications for current and future therapeutic approaches. Clin Cancer Res 2013;19:2842-9.

7. Oberg K. Circulating biomarkers in gastroenteropancreatic neuroendocrine tumours. Endocr Relat Cancer 2011;18: S17-25.

8. Trapé J, Filella X, Alsina-Donadeu M, et al. Increased plasma concentrations of tumour markers in the absence of neoplasia. Clin Chem Lab Med 2011;49:1605-20.

9. Fenoglio PC. Gastrointestinal neuroendocrine/neuroectodermal tumors. Am J Clin Pathol 2001;115 (Suppl 1):S79-93.

10. Zatelli MC, Torta M, Leon A, et al. Chromogranin A as a marker of neuroendocrine neoplasia: an Italian multicenter study. Endocr Relat Cancer 2007;14:473-82.

11. Chromogranin A: is it a useful marker of neuroendocrine tumors? J Clin Oncol 2007;25: 1967-73.

12. Nobels FR, Kwekkeboom DJ, Coopmans W. Chromogranin A as serum marker for neuroendocrine neoplasia: comparison with neuron-specific enolase and the alpha-subunit of glycoprotein hormones. J Clin Endocrinol Metab 1997;82:2622-8.

13. Kloppel G, Clemens A. The biological relevance of gastric neuroendocrine tumors. Yale J Biol Med 1996;69:69-74.

14. Louthan 0. Chromogranin A in physiology and oncology. Folia Biol (Praha) 2011;57:17381.

15. Leung D, Schwartz L. Imaging of neuroendocrine tumors. Semin Oncol 2013;40:109-19.

16. Teunissen JJ, Kwekkeboom DJ, Krenning EP. Quality of life in patients with gastroenteropancreatic tumors treated with [177LuD0TA0,Tyr3] octreotate. J Clin Oncol 2004;22: 2724-9. 